\title{
Las neurociencias en el proceso de enseñanza-aprendizaje para la formación de un director de escena del Instituto de Artes de la Universidad Autónoma del Estado de Hidalgo
}

The neurosciences in the teaching-learning process for the formation of a director scene of the Institute of Arts of the Autonomous University of the State of Hidalgo

\section{Fernando Briones Durán ${ }^{a}$}

\begin{abstract}
:
The following pedagogical reflection tries to locate the importance of the university academic process in the formation of a stage director, locating from the Sciences of the education the differences between theatrical pedagogy and artistic education. To direct didactic research to the influence that neuroscience has had on the formation of new stage artists.
\end{abstract}

Keywords:

Process, training, university, neurosciences, pedagogy, theatrical artistic education

\section{Resumen:}

La siguiente reflexión pedagógica intenta situar la importancia del proceso académico universitario en la formación de un director de escena, ubicando desde las Ciencias de la educación las diferencias entre pedagogía teatral y educación artística. Para dirigir las investigaciones didácticas a la influencia que han tenido las neurociencias en la formación de los nuevos artistas escénicos.

\section{Palabras Clave:}

Proceso, formación, universidad, neurociencias, pedagogía, educación artística teatral

\section{Introducción}

¿Qué es un proceso de enseñanza-aprendizaje teatral para la formación de un director de escena?

Según la RAE (2018) dice que "Proceso es un conjunto o encadenamiento de fenómenos, asociados al ser humano o a la naturaleza, que se desarrollan en un periodo de tiempo finito o infinito y cuyas fases sucesivas suelen conducir hacia un fin específico", es decir, esos fenómenos posiblemente sean un cúmulo de entrecruzamientos biopsicosociales y espirituales que pueden asemejarse al complejo sistema de redes que se generan dentro del cerebro.

Es decir, un proceso formativo de enseñanza aprendizaje, es sistémico y metodológico porque partió de un grupo de estudiosos de la educación que pensaron al hombre respetuoso, tratable, civil. Donde existe una figura llamada docente, el que enseña y otra figura llamada dicente, el que aprende, en lugares instaurados por una sociedad Ilamadas escuelas. El dicente su objetivo es aprender, el propósito del docente es enseñar y la misión de la escuela es proporcionar el ambiente óptimo para que el proceso educativo, como lo menciona el Modelo de la UAEH (2018), tenga "la calidad de los procesos de enseñanzaaprendizaje que tienen lugar en las aulas dependerá, en gran medida, del funcionamiento y organización de la institución". Por lo tanto, un proceso de enseñanza aprendizaje consta de tres elementos fundamentales; el alumno, el profesor y la institución.

En el caso de un proceso de enseñanza-aprendizaje teatral, con énfasis en la dirección escénica resulta ser un poco más complejo porque es integrador, ya que no solo están los elementos biopsicosociales espirituales sino ya abraza ideas filosóficas, antropológicas, biomecánicas, 
artísticas, matemáticas, estéticas, lingüísticas, históricas y geográficas, para generar un nuevo discernimiento.

Por lo tanto este enfoque psicopedagógico teatral constructivista, a través de la enseñanza centrada en el aprendizaje como lo menciona en el Manual de estilos de aprendizajes de la SEP (2004) "obliga a diseñar, incorporar y difundir acciones que lleven a nuestros alumnos a asumir y entender los contenidos de aprendizaje planteados, a través del autoaprendizaje y la responsabilidad compartida", para respetar las diferentes formas que tiene cada alumno de aprender.

Ya visto desde la neurociencias este proceso de aprendizaje será integrador y holístico porque cada alumno será responsable creativamente de reafirmar sus valores éticos y morales en una puesta en escena que determine sus preferencias estéticas.

Por lo tanto un proceso de enseñanza aprendizaje teatral para la formación de un director de escena en la UAEH del instituto de artes, es una experiencia psicopedagógica constructivista dónde todos los elementos que lo conforman estarán integrados sistémicamente a la teoría pedagógica teatral ya existente para la construcción de nuevos modelos de aprendizaje y enseñanza.

\section{Materiales y métodos}

Con relación a este artículo científico el proceso surgió desde la observación cualitativa en las sesiones instructivas llevadas a cabo en el sexto semestre. El alumno universitario del Instituto de Artes de la Universidad Autónoma del Estado de Hidalgo, sumergido en un desánimo intelectual, por la confusión de los conceptos; subjetivos, arbitrarios y determinantes de otras áreas de estudio escénicas y con relación a su vida social y personal, obstaculizaban su imaginación y creatividad.

El uso de la pedagogía freiriana y chejoviana ya aplicados a procesos de enseñanza aprendizaje anteriores, reafirmados en mis tesis de licenciatura y maestría en la formación informal, permitieron entonces en el séptimo semestre, acercarme a las neurociencias a través de estudios sobre metodología y diplomados en educación, para ubicar estrategias didácticas más objetivas y concretas que permitieran al alumnos adentrarse, y hacer consciencia de su conocimiento universitario para el beneficio de su formación como director de escena.

El objetivo; despertar la imaginación creadora y critica, reafirmando sus valores éticos, morales y estéticos es su puesta en escena de octavo semestre, último de su formación universitaria. Que considerada como una tesis, el proceso despertó la inquietud práctica, es decir, el alumno con una actitud alegre, propositiva, autosuficiente, y autodidacta generó su producción, gestión y difusión del espectáculo final de titulación.

Considerándome durante todo el proceso como un Coach artista educativo, que a través de la dialogicidad pedagógica y respetando nuestras individuales ontológicas, integramos al proceso nuestra experiencia para desentrañar un fin común; la génesis de estrategias didácticas con base en la Neuroeducación para la formación de un director de escena.

\section{Desarrollo}

\section{Pedagogía teatral}

Existen en el mundo una cantidad nada despreciable de artistas que no necesariamente se formaron como pedagogos, y que han sugerido desde su particular percepción de vida, la visión del teatro y lo que ellos pensaron debía ser un actor o un director de escena. En las escuelas universitarias donde se forman los actores y directores de escena la mayoría de estos artistas que se dedican a dar clases, supuestamente, parten de los estudios de otro actor ruso del S. XIX Constantin Stanislavski, para justificar su pedagogía teatral, pero sin una claridad didáctica, ya que Stanislavski no menciona como se enseña sino que categorías necesita el actor para sustentar su trabajo vivencial.

Al respecto Serrano (2017) menciona que "Stanislavski no dejo ni un método ni un sistema, lo que dejo es un cuaderno de apuntes con numerosas anécdotas de las cuales uno puede deducir un montón de problemas de tipo pedagógico, de tipo didáctico pero no hay un sistema ni una pedagogía". Y que hasta la fecha muchos no se han solucionado.

Esto ha traído supuestamente, como consecuencia, utilizar el concepto pedagogía teatral, de manera muy arbitraría. Se le llama pedagogía teatral al que enseña, al que habla de los teóricos de fin de siglo, el que da clases en la universidad, al que trabaja con presos, con niños y adolescentes etc. No hay duda que todo esto tiene que ver con los tipos, niveles y metodologías de una educación teatral. Basta que tengas más de dos años dando clase para se diga que ya tiene su pedagogía teatral para enseñar.

Por tal motivo ubicar los conceptos siempre será necesario para comenzar a teorizar. Porque como lo menciona Veites (2013) "se establece lo que pueda ser la pedagogía teatral, considerando las manifestaciones diversas de lo que cabría denominar educación teatral, pues ni esta comienza en la educación primaria ni termina 
en la formación superior" es decir si existe una diferencia conceptual entre los fines y que en los procesos de enseñanza aprendizaje universitario es muy peligroso confundir.

La educación surge y se logra en todos lados y se relaciona con la educación artística que se desarrolla solo en el interior de un salón de clase, pero que el alumno cataloga y está atento a las manifestaciones profesionales de sus profesores, a los performance, ritos y mitos cotidianos, a las culturas de sus comunidades, a las ideologías de sus familias. La educación artística teatral no termina, porque todas esas percepciones están siendo organizadas en el interior del cerebro que es el que siempre está aprendiendo.

Por lo tanto la pedagogía teatral como ciencia, para estudiar la educación artística teatral, necesita de las Ciencias de la educación para pensar y conformar al artista del futuro, triangular las teorías pedagógicas, las pedagogías de la libertad y las experiencias doxas del docente, posiblemente sean procedimientos metodológicos para acceder a las profundidades cognitivas de un proceso en la formación de un director de escena para una educación artística teatral más objetiva, científica e innovadora, al respecto Nassif (1975, p. 3) menciona que:

"[...] una cosa es la pedagogía y otra la educación; una cosa es el objeto y otra, la ciencia que de él se ocupa... En otras palabras: la pedagogía es la disciplina, el estudio o el conjunto de normas, que se refieren a un hecho o a un proceso o actividad, la educación. (Citado en Veites, 2017, pág. 1523).

Por lo tanto la pedagogía teatral son las tesis o el conjunto de normas que guían las didácticas de una educación artística teatral. Porque siguiendo los pensamientos de Veites (2017) no es lo mismo la Pedagogía Teatral entendida como una teoría general de la educación teatral, que las didácticas de la expresión dramática, de la interpretación o de la dirección de actores y actrices". Una teoriza y la otra aplica. Forzar solo el pensamiento a memorizar términos de las pedagogías teatrales $\mathrm{y} / \mathrm{o}$ seguir fielmente las interpretaciones que los artistas hacen de las mismas, solo ha dado como resultado, supuestamente, una gran confusión y deserción escolar.

Por lo tanto la pedagogía teatral tiene que ser seleccionada rigurosamente en el planteamiento de planes de estudio y la selección que hace el docente de los temas para aplicarlos. Por ejemplo; el muy citado y confuso término "vivencial" para realizar acciones físicas verdaderas que los actores y directores en funciones docentes aplican sin ningún rigor científico a la formación de un actor y en el cual el alumno debe relacionar los sentimientos de los personajes con aquellos personales que los han atormentado, solo ha llevado a muchos alumnos a una gran depresión, enojo y soledad por la falta de una didáctica debido al enredo entre pedagogía teatral y educación artística teatral universitaria.

\section{Educación artística teatral universitaria}

Educar significa según la RAE (2018), "Dirigir, encaminar, doctrinar. Desarrollar o perfeccionar las facultades intelectuales y morales del niño o del joven por medio de preceptos". Es decir dejarlo ser para después darle forma de acuerdo a los ideales de una sociedad a través de una educación palabra y acción muy compleja que ahora tiene significados múltiples debido a la existencia de muchos tipos, informales, no formales, formales y un gran etcétera dirigidas a todo el sector de una población desde la educación básica hasta la superior. Desde la casa hasta la integración a los grupos de pertenencia.

El proceso cognitivo de sus integrantes se expande a través de los procesos de socialización. En un intercambio de valores éticos y morales los individuos se educan entre ellos para aprender a pensar, a hacer y sobre todo a ser, para el correcto funcionamiento dentro de la sociedad seleccionada.

Sin embargo la educación dentro de una universidad de arte teatral por supuesto es compleja y sublime, pero más integral, porque está determinada por metas, fines y objetivos. Es compleja por la gran cantidad de epistemologías que adquiere el individuo y sublime porque constituye en su educación todas las artes, clásicas, modernas y contemporáneas. Esto hace que mantener la cultura de origen dentro de una formación universitaria es una constante lucha de los individuos para existir por el gran conductismo que rigen los sistemas educativos.

Este conflicto tendría que desaparecer a través de una educación artística teatral constructivista que permita a los alumnos seleccionar sus propias formas de aprender. Aplicar las correctas metodologías a la educación para descubrir sus propias inteligencias y entender la gran cantidad de signos; lingüísticos, corporales, verbales emotivos que perciben diariamente en su proceso de enseñanza-aprendizaje, es el objetivo de un docente. Por lo tanto, la educación artística teatral universitaria, se refiere a la correcta utilización de pedagogías teatrales integradas a la gran cantidad de didácticas y estrategias que existen en las ciencias de la educación para poder aplicar las teatrales que sin duda apoyarían el proceso formativo de un alumnos y profesor. 


\section{Docente, maestro, profesor, artista o Gurú}

Parecería que los conceptos que definen a un docente universitario que se dedica a la formación de teatreros están inmersos en una maraña de significaciones; es un tutor o una mamá dando consejos de vida a los alumnos, tal vez un psicoanalista que determina la enfermedad de los dicentes por la actitud de sus acciones, o mejor dicho un Guru que fue iniciado en el teatro chamánico y ahora da clases para seguir pasando la información divina a través de cánticos y rezos, o finalmente un fiel seguidor de un gran maestro donde ahora él o ella ha sido designada para continuar con sus enseñanzas.

Un docente según el Modelo Educativo de la UAEH (2018) "es la persona a quien la Universidad encomienda la realización de tareas sistemáticas relacionadas con los procesos de formación, capacitación y adiestramiento que conforman los programas académicos y actividades dedicadas a la educación de los estudiantes" (Pág. 86). Es decir, es una persona especializada en el manejo de técnicas de estudio, que sabe aplicar metodologías de enseñanza y aprendizaje, y finalmente en el caso de la educación artística universitaria, esta persona sabe diferenciar entre su yo artista y su yo docente.

Las dos profesiones tiene fines diferentes: El artista y el profesor o maestro, (no se refiere al grado de estudio) no se contraponen pueden ser integrales. Aunque el docente tiene funciones primordiales según la escuela, instituto o universidad donde se encuentren, el artista no, pero puede organizarse para combinar esta dos actividades. Sin embargo algo que los diferencia es que el profesor debe tener una vocación de servicio, gusto por la docencia, interés por la investigación y es aquella persona que esta decido a servir al otro, sacrificarse por que los otros logren su vocación, Contreras (2015) define lo siguiente:

\begin{abstract}
El término docente es polisémico se usan como sinónimos del mismo las siguientes palabras: pedagogo, instructor, formador, educador, enseñante, adiestrador, maestro, didáctico, académico, normativo, purista, clásico, culto, asesor, consejero, facilitador, promotor, orientador, coordinador, consiliario, tutor, gestor, mentor, guía, gurú, mediador y conductor, entre otras.
\end{abstract}

Es claro que todas estas definiciones, responden a característica diferentes sin embargo en lo que todos coinciden es que su objetivo es enseñar. Por lo tanto también el docente universitario enseña pero tiene la obligación de usar métodos, técnicas instrumentos y procedimientos de la docencia, la investigación que pueda ordenar el proceso de la enseñanza-aprendizaje.

Por otro lado el artista teatral, ya sea actor, director o dramaturgo no necesariamente tiene que saber enseñar, pues no estudio para eso, por lo tanto es casi una obligación integrarse a cursos sobre pedagogía que le permitan ejercer este carrera al respecto Contreras (2015) menciona que:

La docencia va más ligada al cambio de la persona que recibe la enseñanza que a la capacidad de uno de expresar un concepto. Muchos hemos pasado por experiencias universitarias en que abogados, arquitectos 0 médicos intentan dar cuenta de su saber, siendo incapaces de entregarlo en forma clara y sencilla. (párr. 1)

Por lo tanto el docente universitario dentro del recinto escolar, es una paradoja, porque es y no es artista. Es profesor, capaz de entender que su actividad docente, es un asunto amoroso, erótico, creativo y de mucha paciencia, porque este artista-docente conoce las didácticas dentro de los procesos de enseñanzaaprendizaje, que son sorprendentemente lentos y que ahora las neurociencias ya se encargan de estudiarlos.

\section{Una neurona creativa}

Las neurociencias en el proceso de enseñanzaaprendizaje para la formación de un director de escena del Instituto de Artes de la Universidad Autónoma del Estado de Hidalgo, parte de la idea de saber que el cerebro humano se está transformando, ya es otro. "Según estudios realizados por científicos de la Universidad de Missouri, en Estados Unidos, supone la primera comprobación empírica y rigurosa de la hipótesis del predominio de la presión demográfica en el desarrollo de nuestro cerebro". (Martínez. 2018. párr. 1) Descubrir que el ser ya no explica su existencia a través de los ritos, mitos y leyendas como en la antigüedad, ni tampoco le da gran importancia a la existencia de lo divino, ni mucho menos a explicar su estancia en la lucha de clases. Es obvio pensar que todo este cambio en la plasticidad cerebral, "La plasticidad cerebral se refiere a la capacidad del sistema nervioso para cambiar su estructura y su funcionamiento a lo largo de su vida, como reacción a la diversidad del entorno". (Kolb, B., Mohamed, A., \& Gibb, R. 2010. párr. 1) se debe a la gran cantidad de humanos que ocupamos este planeta.

La economía sucumbió en algún momento en el siglo pasado, su efecto fue fundamental y su inmersión en el mercado laboral y sobre todo en los sistemas educativos 
del mundo resulto globalizante. "Este nuevo ciclo ha sido denominado sociedad de la información, debido a que es la información la que ahora dirige la economía global que está surgiendo. (Rodríguez. 2014; 4). Siguiendo las ideas de Martínez (2018), "en los últimos dos millones de años, el cerebro humano se ha triplicado", la estructura biopsicosocial espiritual del hombre se sigue transformando, su cultura, el clima y sobre todo la gran cantidad de seres humanos que se relacionan en una sociedad por demás competitiva que a través del internet y las redes sociales ahogada de información ha creado en el cerebro homínido el ambiente necesario para que este órgano biológico que controla y dirige nuestro comportamiento se transforme a sí mismo.

Todo esto nos lo explica Sáez (2014) citando a David Bueno, profesor de genética de la Universidad de Barcelona, especializado en la formación del cerebro y divulgador científico. "ha abierto una nueva etapa para poder conocernos a nosotros mismos, para entender mejor cómo funcionamos y aplicar ese conocimiento a áreas tan diversas como la economía, la cultura y la educación".

Los estudios que se hacen en la educación universitaria y las neurociencias no se alejan de esta transformación del cerebro homínido desde el enfoque de la transdisciplinariedad y los estilos de aprendizaje, en su estudio Vallejo y Sanandrés (2016) mencionan que:

Para la educación superior es importante las investigaciones realizadas desde la visión neurocognitiva, porque es necesario docentes y estudiantes conozcan sus estilos de aprendizaje, la forma de aprender y su manera de estudiar basada en esas diferencias que es más eficaz para cada persona. (Pág. 22)

Desde la formación clásica hasta las muy transformadoras pedagogías de la Nueva escuela, las Ciencias de la educación y sobre todo las pedagogías de la libertad han permitido avanzar en la transmisión de los valores éticos y morales a las nuevas generaciones a través de didácticas que ya se preocupaban por la función holística-cognitiva de los estilos de aprendizaje.

\section{La neurociencia sensible en la formación de un director-actor}

El proceso biopsicosocial en la enseñanza-aprendizaje universitaria para encontrar las didácticas que estimulen los sentidos y lograr percepciones más holísticas que determinen un comportamiento estético en la formación de un director de escena. Al respecto Calle (2015) en el resumen de su artículo Cerebro y sujeto. Una producción biopsicosocial nos indica que:
Este documento constituye una reflexión sobre el puente entre la neurociencia y el comportamiento social. Temas como el cerebro, la mente, la percepción y el comportamiento se revisan bajo una visión transdisciplinaria. De esta manera, la antigua perspectiva de la división objetiva y subjetiva de la comprensión de la mente, ha sido sustituida por una idea holística en la cual las conexiones cerebrales, la realidad social y el ser, son articuladas al mismo tiempo. (pág. 100)

Sin duda la creación de artistas y las escuelas donde se forman no se alejan de esta de esta observación científica. Al respecto Fons (2015) nos dice "Y como los avances actuales en el campo de las neurociencias afectan de manera significativa a las artes escénicas, y a la comprensión del proceso creativo del actor en particular", podemos deducir que un actor del Instituto de Artes y desde esta visión holística también puede ser director, dramaturgo, escenógrafo, tramoyista, festivo, amante y bailador, que sin dudarlo y ante el avance de la tecnología cibernética, el reguetón y las tan sobadas, sorprendentes, graciosas, impresionantes, enajenantes, perturbadoras y tremendas redes sociales su cerebro ya se transformó.

La línea de investigación, es saber qué acciones, ejercicios y prácticas, es decir, qué tipo de didáctica se tendría que aplicar desde las neurociencias para formar a un artista integral, Fons (2015) menciona que "la comprensión del fenómeno teatral desde la perspectiva de la neurociencia cognitiva para plantear una nueva teatrología pluridisciplinar y experimental" que nos permita como docentes y alumnos despertar la creatividad y la innovación en los procesos de aprendizaje y enseñanza, al respecto Campesinos (2013) deduce en el encuentro de Pedagogía teatral :

Mientras esto ocurre, tiene lugar el importantísimo desarrollo del proyecto Zero, que permitió repensar el aprendizaje artístico desde una perspectiva cognitivista y entender la inteligencia humana como un complejo proceso de articulación con el mundo y de construcción multidimensional de significados. Así, no habría una única inteligencia, sino una variedad de procesos que se caracterizarían por su predominio en campos diferenciados (espacial, musical, lingüístico, matemático, etcétera).

Las experiencias de un profesor-artista teatral, y las expectativas de un alumno en formación se podrían integrar en un solo discurso estético a partir de un modelo neurocientífico pedagógico y teatral. Caminar juntos en 
la extraordinaria y misteriosa creación artística escénica dentro de estos nuevos ambientes de aprendizaje a partir de una metodología científica, la formación de un director de escena, podría dar las bases para la creación de una didáctica científica y representacional. Al respecto Veites (2013) menciona que el:

Conocimiento de las teorías de la educación, del aprendizaje y de la psicología del desarrollo. Conocimiento y práctica de procesos de diseño y desarrollo curricular, planificación didáctica y elaboración de materiales para la práctica educativa y la animación teatral. Conocimiento de los principios teóricos y metodológicos que orientan el diseño, desarrollo y evaluación de procesos de enseñanza-aprendizaje y de programas de animación teatral, en función de diferentes espacios, tiempos y beneficiarios. Conocimiento, análisis y práctica de métodos y estilos de enseñanza, aprendizaje y animación. Conocimiento básico de los aspectos básicos de la historia de la educación y de la animación teatral. Estudio de casos y análisis de buenas prácticas. La investigación aplicada a la educación y la animación teatral. Políticas de garantía y gestión de calidad.

\section{Conclusiones}

\section{El neuroteatro}

Es un supuesto comprender entonces que para que se pueda realizar un estudio sobre las neurociencias y el teatro, necesariamente el conocimiento de las Ciencias de la Educación nos dará la posibilidad de ubicar; la pedagogía teatral de una educación artística teatral, para proponer una Andragogia de la formación del director de escena, para que la misma disciplina cuente con un lenguaje propio donde se pueda reconocer los errores semánticos del conocimiento científico del proceso creativo y así evolucionar hacia innovaciones estéticas en la culminación y/o egreso de un alumno del Instituto de Artes con énfasis en dirección.

Universitario formado a través del Modelo* Educativo de la Universidad Autónoma del Estado de Hidalgo que determina una responsabilidad deontológica de la universidad con sus alumnos para que "se sientan satisfechos del deber cumplido; rijan sus actos por los

\footnotetext{
* Un modelo es, sobre todo, algo con qué compararse, un paradigma por alcanzar y una guía práctica para orientar el comportamiento de quienes participan en los hechos educativos y administrativos de la institución... ( ) Bajo estas premisas, el Modelo Educativo se diseña como sistema que confluye en un gran proceso central, el
}

dictados de la moral y el respeto a las diferencias ajenas; y actúen con una disciplina derivada del convencimiento, no del sometimiento o la injusticia, a lo cual deberán oponerse" (Modelo educativo).

Un alumno artista que posea altas competencias estéticas en el desarrollo profesional de su actividad para el mejoramiento biopsicosocial de la comunidad de origen, para coadyuvar en la búsqueda de un teatro mexicano determinado por un proceso científico educativo que se represente como una conducta emocional diferente al status quo.

Dicho en otras palabras, supuestamente al aplicar una didáctica neurocientífica al proceso de aprendizaje para formar a un director de escena del Instituto de Artes, el producto tendrá que ser diferente a lo ya establecido porque la enseñanza se generó a partir del conocimiento científico. Cerebro, teatro y cognición, muy relacionadas con los estados emotivos del aprendizaje. Andragogia y Teatrología binomio sistémico que nos permitirá realizar una gran cantidad de entrecruzamientos al igual que sucede dentro de la mente, directamente proporcionales a las miles de corrientes eléctrico-nerviosas que llevan información biopsicosocial-espiritual y cruzan infinidad de veces por los axones, donde "las funciones de la corteza cerebral se producían por una actividad cortical global. Nacía, ahora con los axones, la teoría de una enorme red nerviosa difusa interaxonal por la que fluían los impulsos nerviosos.

Por tanto, podríamos decir según Fresquet. (2015) Se creía en un tejido nervioso que era una red continua, bien dendrítica (Gerlach), bien cilindroaxil (Golgi)", hasta reconstruirse en miles de sinapsis creadoras que generarían experiencias ricas e innovadoras. Innovadoras porque cada día, cada hora, cada minuto, cada segundo la cognición se mueve en unidades perceptivas, creando conocimientos, permítaseme la expresión hipotética, sapiencias microcelulares que cambiarían la conducta del artista teatral. Y rica porque al respecto García (2018) nos dice que una experiencia rica:

Se refiere a experiencia de aprendizaje que propongan desafíos y retos; que proporcionen guías, pero no soluciones; que ofrezcan pautas y rutinas para la creación de pensamientos

denominado "proceso educativo", alineado por la sinergia y fundamentado en el marco filosófico que le confiere orientación humana, histórica y política, generando la fortaleza de la visión universitaria que nutre los valores comprometidos con el desarrollo científico, tecnológico y humano. 
ordenados, que ayuden al cerebro a organizar el caos. Unas experiencias que hagan aflorar preguntas, interrogantes e hipótesis a nuestro alumnado, que les inciten a buscar soluciones; que respondan a cuestiones reales; que correspondan a problemas múltiples y flexibles soluciones y caminos; que les obliguen a interaccionar con otros y a crear equipo. Ricas son las experiencias que promueven pensamiento científico, pensamiento lateral y pensamiento creativo, que movilicen fundamentalmente pensamientos de orden superior, que les obliguen y enseñen a pensar.

Es decir una pedagogía teatral rica en experiencias didácticas para una educación artística universitaria alegre, amorosa, que ría, que cante, que incluya, y sobre todo que sienta; porque a fin de cuentas el viejo axioma y paradigma del artista que sufre y se desgarra dolorosamente por al arte o de ese teatro con relación a lo vivencial o emotivo del ser profanado... ha caído por el hoyo de la posmodernidad, alejada de los estereotipos convencionales del maestro "guru", del docente artista impositivo y transgresor o de aquel maestro vacacionista que en nombre de su arte, beca o situación popular no llega a clase o deja un suplente alumno.

Eso ya no existe para esta nueva educación neurológica cognitiva teatral. Para estos nuevos seres/alumnos nacidos de lo cuántico y cibernético el neuroteatro, el teatro y cerebro serán las nuevas líneas de investigación que ahora aplicaremos a la formación de un director de escena. Retomando la pregunta de García Pérez ¿Es posible desarrollar un aprendizaje basado en el cerebro? (2017). Yo agregaría, ¿Es posible desarrollar un aprendizaje teatral basado en el cerebro en el proceso de enseñanza-aprendizaje para la formación de un director de escena del Instituto de Artes de la Universidad Autónoma del Estado de Hidalgo? He ahí el camino.

Entonces para poder encuadrar el estudio, supuestamente, podemos argumentar que se trata de generar una educación artística teatral que ha partido de una pedagogía primeramente plasmada en los objetivos del Modelo Educativo mencionada anteriormente. Llegamos así a nuestra pedagogía artística determinada por la oferta educativa del Instituto de Artes "ofrecer un lugar ideal para desarrollar tu talento y tus habilidades, cumpliendo así con la vocación de nuestra Universidad: formar a las mujeres y hombres que plasmen en la realidad la riqueza de nuestro espíritu". (2018). Hasta llegar a los objetivos de la pedagogía teatral de la licenciatura de Arte Dramático ya determinados en la información que se da en la página del Instituto (2018);

Formar licenciados Arte Dramático, artistas escénicos creativos capaces de enfrentar cualquier exigencia teatral y escénica a través de referentes teóricos y técnicos propios de la disciplina, con el propósito de propiciar experiencias estéticas entre ellos y los espectadores. Con un alto nivel de competencia profesional, dotados de sólidos conocimientos teóricos y prácticos; que desarrollen a través de la creación coreográfica, la docencia y la ejecución, habilidades creativas, de reflexión y de autocrítica, disposición para el trabajo en grupo, valores éticos y estéticos.

Es decir formar, educar, enseñar, adiestrar, dirigir y desarrollar requiere de didácticas, de estrategias docentes, de pedagógicas escénicas para que el director teatral del Instituto de Artes se forme, ya no en un sentido místico, ni religioso sino a través de un asunto neuronal, científico y metodológico que le permita nuevamente redescubrir el espíritu del hombre contemporáneo.

Porque retomando las palabras de Bretel (2015) "tenemos en nuestros hombros una de las máquinas más poderosa que existe y sin embargo vino sin manual" en este sentido poder crear una didáctica, ¿un manual?, que permita al docente a enseñar los misteriosos métodos y metodologías pedagógicas del arte teatral que forman a un director de escena en el IA. Ya existen estudios sobre el tema; "Teatro y neurociencia; el proceso creativo del actor desde la neurofisiología de la acción" discutidos por Martín B. Fons Sastre, desde las neuronas espejos, Gabriele Sofía de la Universidad de Grenoble "Las acrobacias del espectador, neurociencia, teatro $y$ viceverse", Miguel Rivagorda de la UCM "El espectadorinterprete, ¿Por qué a mí me ha gustado y a ti no? Cognición enactiva" y Elena Martín, Co-founder y CEO de Sociograph, "Marketing Science y el impacto en las artes escénicas" descubiertas en una conferencia de la RESADMADRID Publicado el 15 nov. 2017*. Así mismos un texto de Gittih Ariela Sanchez Padilla; "teoría de la complejidad: Neurociencia y Biodanza, donde en cada investigación y especialidad se certifica y comprueba la manera en que estos estudios neurocientífico impactan en la creatividad y desarrollo de sus áreas, mas no, como se enseñan y como se aprenden.

Sin dudarlo a nivel educación universitaria ya se aplican en otras disciplinas, ahora será el asunto de esta línea

\footnotetext{
* Vélez Julio (2015) Teatro-neurociencia. ITEM. Madrid. RESAD

https://www.youtube.com/watch?v=3VXdiSu5i-A\&t=794s
} 
de investigación emergente, saber cómo se utilizarían para la formación universitaria de un director de escena en el Instituto de Artes.

\section{Referencias}

Fons S. Martín Bienvenido. (2015) Teatro y neurociencias: el proceso creativo del actor desde la neurofisiología de la acción. Artículos. Acotaciones. Escola Superior d'Art Dramàtic de les Illes Balears (ESADIB)

Calle Sandoval, Diego Alejandro. (2015). Cerebro y sujeto: una producción biopsicosocial. Pensamiento Americano Vol. 8. No. 15. Universidad San Buenaventura, Medellín. Corporación Universitaria Americana. Barranquilla, Colombia. Recuperado: http://www.coruniamericana.edu.co/publicaciones/ojs/index.ph p/pensamientoamericano/article/view/329

Kolb, B. Mohamed, Gibb A., R. (2010). Plasticidad Neuronal y Cognición. Estructura y organización. CogniFit. Revista de Trastornos de la Comunicación. Recuperado https://www.cognifit.com/es/plasticidad-cerebral

Contreras S. Georgina. (2015) ¿Ser o no ser... docente, educador, profesor o maestro? México. Iberciencia. Comunidad de Educadores Iberoamericanos para la Cultura Científica. OEI. La vocación docente es el conjunto de intereses, necesidades, aptitudes, ideales... (Párrafo https://www.oei.es/historico/divulgacioncientifica/?Ser-o-noser-docente-educador-profesor-o-maestro

Fresquet L José. (2015). Aparato, corpúsculos, ley de Golgi. Instituto de Historia de la Ciencia y Documentación (CSICUniversitat de Valencia). Epónimos Médicos. Historia de la Medicina. https://www.historiadelamedicina.org/Golgi.html Recuperado:

García P. José Blas. (2017). La neurociencia aplicada a la educación en 10 consejos. España. Facultad de educación de la Universidad de Murcia. Web del maestro WMCMF. Recuperado: http://webdelmaestrocmf.com/portal/laneurociencia-aplicada-la-educacion-diez-consejos/

Bretel L. (2015) ¿Cómo aprende el cerebro? Aprende con Neuroeducación.

https://www.youtube.com/watch?v=7J17gs67L5k\&t=765s educación pública. Subsecretaría de educación media superior. Dirección general del bachillerato Dirección de coordinación académica. (Pág. 2)

Martínez, Yaiza. (2018). El cerebro humano se desarrolló gracias a la competitividad social. Tendencias científicas. Tendencia 21. Recuperado. https://www.tendencias21.net/El-cerebrohumano-se-desarrollo-gracias-a-la-competitividadsocial_a3413.html

Proceso. (s.f).En Wikipedia Recuperado 11 abr 2018 a las 00:31. https://es.wikipedia.org/wiki/Proceso

Real Academia Española. (2018). Asociación de academias de la lengua española. RAE.es. Felipe IV, 4 - 28014 Madrid. Recuperado. http://dle.rae.es/?id=UFbxsxz

Rodríguez Vite, Higor. (2014) Ambientes de aprendizaje. Ciencia Huasteca. Boletín científico de la Escuela Superior de Huejutla. Publicación Semestral No.4. Julio 2014. Universidad Autónoma de Estado de Hidalgo. Secretaría de Desarrollo Internacional.

Santos C. Georgina (2015). ¿Ser o no ser... docente, educador, profesor o maestro? Iberoamérica Divulga. México. IBERCIENCIA. Comunidad de Educadores Iberoamericanos para la Cultura Científica. Recuperado https://www.oei.es/historico/divulgacioncientifica/?Ser-o-noser-docente-educador-profesor-o-maestro

Sáez, Cristina (2014). Neuroeducación, o cómo educar con cerebro. Periodismo reflexivo sobre ciencia, arte, tecnología y pensamiento. En Revista Quo de México. Recuperado: https://cristinasaez.wordpress.com/2014/10/06/neuroeducacion -o-como-educar-con-cerebro/

Serrano, Raúl (2017). Una pedagogía teatral después de Stanislavski. Facultad de Arte Unicen. Congreso Internacional de Artes Escénicas. Recuperado: https://www.youtube.com/watch?v=b8h_T70ONGc\&t=2313s

Universidad Autónoma del Estado de (2018) Modelo Educativo de la UAEH. II- Aprendizajes de calidad y atención a la diversidad: ejes prioritarios de las políticas educativas. Inciso C, párrafo 1 . Pp. XXIV, XXV. 
Universidad Autónoma del Estado de Hidalgo. (2018). Oferta $\begin{array}{lll}\text { educativa. } & \text { (Párr. } & \text { 2). }\end{array}$ https://www.uaeh.edu.mx/campus/ida/oferta.html

Universidad Autónoma del Estado de Hidalgo. (2018). Licenciatura en Arte Dramático. School of Arts. Información para licenciatura en arte dramático. (Párr. 1) Recuperado: https://www.uaeh.edu.mx/campus/ida/oferta/licenciaturas/lic_a rtedramatico.html

Vallejo Altamirano, Danilo, Sanandrés Álvarez, Luis (2016). Neurociencia en la Educación superior. Desde el enfoque de la transdisciplinariedad y estilos de aprendizaje. Revista Científica INVESTIGAR. Segunda Edición.

Veites G. Manuel Francisco. (2013). La construcción de la pedagogía teatral como disciplina científica. REP. Revista española de pedagogía. Año LXXI. No. 256.

Veites F. Manuel. (2017). La Pedagogía Teatral como Ciencia de la Educación Teatral. Universidad de Vigo (UVIGO), Pontevedra - España. Educação \& Realidade, Porto Alegre, v. 42, n. 4 , p. $1521-1544$ 